\title{
Health care resource utilization and costs associated with advanced or metastatic nonsmall cell lung cancer in the United States
}

\author{
Xinke Zhang, PhD; Daniel C Beachler, PhD, MHS; Elizabeth Masters, MS, MPH; Frank Liu, PhD; Mo Yang, PhD; \\ Jade Dinh, MPH, MBA; Aziza Jamal-Allial, PhD; Francesca Kolitsopoulos, MPH; and Francois-Xavier Lamy, MPH
}

\section{What is already known about this subject}

- Previous studies have highlighted that the economic burden associated with the treatment of nonsmall cell lung cancer (NSCLC) is substantial.

- In recent years (2015 onward), the treatment landscape for advanced NSCLC has drastically changed with the approvals of immune checkpoint inhibitors (ICls).

- A limited number of studies are investigating the economic impact of ICls on the treatment of NSCLC; however, the overall economic burden of advanced NSCLC in recent years is unclear.

\section{What this study adds}

- This study assessed health care resource utilization (HCRU) and costs associated with advanced NSCLC in the United States from 2010 to 2019, which includes the introduction of $\mathrm{ICl}$ in clinical practice.

- Outcomes from this study showed that NSCLC is still associated with considerable HCRU, which is driven primarily by outpatient visits.

- Similarly, the economic burden associated with NSCLC remains substantial. While the introduction of ICls may have been the driving factor for increased costs, inpatient services, other outpatient services, and pharmacy services still contributed to the majority of costs.

\section{Author affiliations}

Xinke Zhang, PhD, EMD Serono, Billerica, MA. Frank Liu, PhD, and Mo Yang, PhD, EMD Serono, Rockland, MA. Daniel C Beachler, PhD, MHS; Jade Dinh, MPH, MBA; and Aziza Jamal-Allial, PhD, HealthCore Inc., Wilmington, DE. Elizabeth Masters, MS, MPH, and Francesca Kolitsopoulos, MPH, Pfizer, New York, NY. Francois-Xavier Lamy, MPH, the healthcare business of Merck KGaA, Darmstadt, Germany.

\section{AUTHOR CORRESPONDENCE:}

Daniel C Beachler, 302.230.2089;

dbeachler@healthcore.com

J Manag Care Spec Pharm 2022;28(2):255-65

Copyright $\odot 2022$, Academy of Managed Care Pharmacy. All rights reserved.

METHODS: Patients with locally advanced or metastatic NSCLC who initiated first-line (1L) systemic treatment between January 1, 2010, and June 30, 2019, were included from the HealthCore Integrated Research Database using a previously developed claims-based predictive model algorithm. Mean total HCRU and costs and mean per-person-peryear (PPPY) HCRU and costs were estimated for 2 follow-up periods: the time during the entire follow-up period and the time during the $1 \mathrm{~L}$ treatment period. Distribution of treatment classes (defined as chemotherapy, ICls, targeted therapies, and others) were also analyzed by index year.
RESULTS: 27,257 patients met the eligibility criteria and were included in the analysis. The mean duration of follow-up for all patients was 16.6 months (median 10.6 months), and the median time to discontinuation of $1 \mathrm{~L}$ treatment was 2.8 months. The number of outpatient visits accounted for the majority of HCRU across the entire study follow-up (mean 97.7 in total and 147.1 PPPY) and for the $1 \mathrm{~L}$ treatment period (mean 46.3 in total and 167.5 PPPY). The total mean cost across the entire study follow-up was $\$ 158,908$ ( $\$ 250,942$ PPPY). For the $1 \mathrm{~L}$ treatment period, the total mean cost was $\$ 72,760$ ( $\$ 271,590$ PPPY). Total mean outpatient costs for systemic anticancer 
treatment were $\$ 61,797$ for the entire study follow-up ( $\$ 85,609$ PPPY) and $\$ 27,138$ during the $1 \mathrm{~L}$ treatment period $(\$ 92,412 \mathrm{PPPY})$. Total costs increased over the study duration, which were mainly due to increasing outpatient costs for systemic therapy. In both follow-up periods, inpatient costs, other outpatient costs (nonsystemic therapyrelated costs), and pharmacy costs remained relatively stable but still accounted for more than $60 \%$ of the total costs. Analysis of treatment classes over time showed that chemotherapy was the most frequently used treatment, regardless of line of therapy. A trend was observed for increased ICI use from 2015 onward.

CONCLUSIONS: Despite the improvement in treatment options, a high economic burden associated with the treatment of NSCLC still exists. The total costs have been increasing, mainly driven by outpatient costs for systemic therapy, which might reflect the greater use of $\mathrm{ICls}$ for advanced NSCLC. Costs for inpatient services, other outpatient services, and pharmacy services remained stable but still accounted for the majority of the economic burden. Further studies are required to assess the impact of innovative treatments on the disease management costs of advanced NSCLC.

Lung cancer is the second most common cancer in the United States, contributing to approximately $12 \%$ of all new cancer cases in 2021. The majority of newly diagnosed lung cancer cases are in the advanced or metastatic setting (stage III/IV), for which survival outcomes have been historically poor. ${ }^{1}$ The most common type of lung cancer is nonsmall cell lung cancer (NSCLC), which constitutes approximately $84 \%$ of total cases. ${ }^{2}$

In recent years, there has been a paradigm shift in the treatment landscape of NSCLC from chemotherapy to targeted therapies and immune checkpoint inhibitors (ICIs). Targeted therapies are selected based on histologic subtype (squamous vs nonsquamous) and molecular testing (including presence of EGFR mutations, ALK rearrangements, ROS1 rearrangements, BRAF V600E mutations, NTRK1/2/3 gene fusions, METex14 skipping mutations, and RET rearrangements), while suitability for ICIs is driven by the absence of molecular mutations and programmed death-ligand 1 (PD-L1) status. ${ }^{3}$

Since 2015, various ICIs, as monotherapy or in combination regimens, have been approved for patients without ALK, EGFR, or other biomarker-driven mutations and have become established therapeutic options for the treatment of advanced NSCLC. ${ }^{3,4}$ Pembrolizumab and nivolumab (anti-programmed cell death-1 [anti-PD-1] monoclonal antibodies) monotherapies were approved by the US Food and Drug Administration (FDA) in 2015, followed by atezolizumab (anti-PD-L1 monoclonal antibody) monotherapy in
2016 as second-line treatment for advanced/metastatic NSCLC. ${ }^{4-6}$ Durvalumab (anti-PD-1 monoclonal antibody) monotherapy was also approved by the FDA in 2017 for use in the locally advanced setting for patients who did not have disease progression following prior platinum-based chemoradiation. ${ }^{7}$ Pembrolizumab (2016), ${ }^{8,9}$ atezolizumab (2020), ${ }^{10,11}$ and cemiplimab (2021) monotherapies were subsequently approved by the FDA as first-line (1L) treatments for patients with metastatic NSCLC with high PD-L1 expression. ${ }^{12,13}$ More recently, atezolizumab (with or without bevacizumab), pembrolizumab, and nivolumab (with ipilimumab) were also approved by the FDA as $1 \mathrm{~L}$ treatments in combination with chemotherapy. ${ }^{14-23}$

Based on these regulatory approvals, ICIs (as monotherapy and in combination with chemotherapy) have become recommended standard-of-care treatment in National Comprehensive Cancer Network guidelines for patients without ALK/EGFR or other oncogenic tumor driver mutations. ${ }^{3}$ Given the transformation in the treatment landscape in the United States, the economic burden with NSCLC may have changed. Historical studies have shown that NSCLC is associated with a considerable economic burden, and health care resource utilization (HCRU) was driven primarily by hospitalizations for adverse event (AE) management. ${ }^{24,25}$ However, the treatment landscape has evolved drastically since these studies were conducted.

In addition, other recent studies regarding the impact of systemic treatments on HCRU reported shorter follow-up periods, had relatively smaller patient populations, or were conducted in other regions around the world. ${ }^{25-28}$ In order to better understand the economic burden of NSCLC since the introduction of newer treatments, such as ICIs, we performed an observational study of patients with advanced NSCLC from 2010 to 2019. We present HCRU and costs from the largest cohort to date and, to our knowledge, of patients with advanced NSCLC who initiated 1L treatment, including ICIs, in the United States.

\section{Methods}

\section{DATA SOURCES AND STUDY DESIGN}

A cohort of patients with locally advanced or metastatic NSCLC who initiated $1 \mathrm{~L}$ systemic treatment between January 1, 2010, and June 30, 2019, was established from the HealthCore Integrated Research Database (HIRD) using a predictive model algorithm for advanced-stage NSCLC developed by linking the claims data with state cancer registries or HealthCore Integrated Research Environment (HIRE) Oncology data. ${ }^{29,30}$ 
This claims-based predictive model algorithm for advanced-stage NSCLC was developed among the subset of patients with clinical data using least absolute shrinkage and selection operator (lasso) regression, and 20-fold cross validation. ${ }^{24}$ At the chosen probability cutoff to define a case, the algorithm's sensitivity was $80 \%$, its specificity was $83 \%$, and positive predictive value (PPV) was $91 \% .{ }^{24}$

The HIRD is a longitudinal medical and pharmacy claims database of health plan members across the United States. Member enrollment, medical care, outpatient prescription drug use, outpatient laboratory test result data, and health care utilization are tracked for health plan members. HIRE Oncology is a preauthorization program in which clinical data are obtained through physician submissions of intentions to use certain cancer treatments and has shown good agreement with medical records with regard to cancer stage. ${ }^{25}$

Eligibility criteria required patients to be matched to a state cancer registry (Ohio, Kentucky, and New York) or to HIRE Oncology records if they had a confirmed diagnosis of locally advanced (stage IIIa or IIIb) or metastatic (stage IV) NSCLC, or if they met the advanced-stage NSCLC predictive model algorithm threshold criteria for advanced or metastatic disease. Patients also had to have continuous enrollment in the HIRD of at least 6 months prior to the first lung cancer diagnosis in the HIRD. Patients were commercially insured individuals (eg, employer-based or Medicare Advantage, not including Medicaid patients). The index date was defined as the date of diagnosis for advanced disease, determined either as the date that patients met the algorithm threshold criteria for advanced disease or met the cancer registry or HIRE Oncology advanced cancer date for confirmed cases (ie, date of confirmed advanced cancer). A probabilistic linkage with the National Death Index was performed for eligible patients to capture mortality. Patients were followed from their index date to the earliest record of death, disenrollment, or end-of-study period (June 30, 2019).

HCRU was assessed during follow-up, and corresponding costs were estimated. Costs for years prior to 2017 were adjusted to 2017 dollars using the medical care component of the US Consumer Price Index; costs for 2018 and 2019 were not adjusted.

\section{MEASURES OF HCRU AND COSTS}

HCRU included hospitalizations (subsequent hospitalizations were considered new if they occurred $\geq 24$ hours after prior discharge date), outpatient visits (defined using the Berenson-Eggers Type of Service coding system, including office visit evaluations and management, physician services, various procedures, imaging, laboratory and other tests, durable use of medical equipment, medication and related services, physical/occupational/speech therapy, other [preestablished definition]), ${ }^{31}$ and inpatient length of stay. Among the outpatient visits, office visits, emergency department visits, and laboratory tests were also reported separately. Costs were measured as total costs paid by both payers and patients. Costs included inpatient costs, outpatient systemic therapy costs (ie, intravenous cancer therapy costs), other outpatient costs (ie, all outpatient costs outside of systemic therapy costs), and pharmacy costs (eg, costs for oral anticancer drugs such as tyrosine kinase inhibitors).

The distribution of treatments was also summarized by index year; treatments were categorized by class, including chemotherapy, targeted therapies (eg, EGFR tyrosine kinase inhibitors, VEGF inhibitors, PARP inhibitors, ALK inhibitors, ROS1 inhibitors, and HER2 inhibitors), ICI-based therapies (including monotherapy and combinations), and others (defined as chemotherapy rescue/antidote agents [ie, leucovorin], antineoplastic agents as monotherapies and combinations, or cytokine therapies).

\section{DATA ANALYSIS}

Categorical variables were described by counts and percentages. Continuous variables were described by statistics such as mean, standard deviation, median, and interquartile range. Per-person-per-year (PPPY) HCRU and costs were calculated as total HCRU or costs per month across the duration of follow-up (in months) multiplied by 12 months. Total HCRU and costs were defined as the total HCRU and costs over the follow-up period. PPPY HCRU and costs, and total HCRU and costs, were reported by 2 follow-up periods: (1) the time during the entire follow-up period (defined as the period from the start of $1 \mathrm{~L}$ treatment after advanced NSCLC diagnosis to death, disenrollment, or the end of the study period) and (2) the time during the $1 \mathrm{~L}$ treatment period (defined as the period from the start of $1 \mathrm{~L}$ treatment after advanced NSCLC diagnosis to the end of $1 \mathrm{~L}$ treatment, death, disenrollment, or the end of the study period), both with variable follow-up periods across patients (no minimum duration of follow-up; Supplementary Figure 1, available in online article).

The end of $1 \mathrm{~L}$ treatment was inferred based on observed patterns of medication use, which assumed that each cycle was 28 days. The end of $1 \mathrm{~L}$ treatment occurred when at least 60 days between 2 cycles had elapsed, when treatment switches occurred, or when additional treatments were added. ${ }^{32}$ The PPPY HCRU and costs during the entire follow-up period and during the $1 \mathrm{~L}$ treatment period were also summarized by index year from 2010 to 2018 (patients 


\begin{tabular}{|c|c|c|}
\hline Characteristic & \multicolumn{2}{|c|}{$N=27,257$} \\
\hline \multicolumn{3}{|l|}{ Age, years } \\
\hline Mean (SD) & 61.8 & (11.7) \\
\hline Median (IQR) & 62 & $(55-69)$ \\
\hline \multicolumn{3}{|l|}{ Sex, n (\%) } \\
\hline Female & 13,008 & $(47.7)$ \\
\hline Male & 14,249 & $(52.3)$ \\
\hline \multicolumn{3}{|l|}{ US region, $\mathrm{n}(\%)$} \\
\hline Midwest & 7,712 & (29.3) \\
\hline Northeast & 4,658 & $(17.7)$ \\
\hline South & 8,287 & $(31.5)$ \\
\hline West & 5,637 & $(21.4)$ \\
\hline \multicolumn{3}{|l|}{ Index year, n (\%) } \\
\hline 2010 & 2,718 & $(10.0)$ \\
\hline 2011 & 2,640 & $(9.7)$ \\
\hline 2012 & 2,247 & $(8.2)$ \\
\hline 2013 & 2,401 & $(8.8)$ \\
\hline 2014 & 2,725 & $(10.0)$ \\
\hline 2015 & 3,806 & $(14.0)$ \\
\hline 2016 & 3,767 & $(13.8)$ \\
\hline 2017 & 3,194 & $(11.7)$ \\
\hline 2018 & 2,809 & $(10.3)$ \\
\hline \multicolumn{3}{|l|}{ Plan type, n (\%) } \\
\hline Commercial & 21,349 & $(78.3)$ \\
\hline Medicare Advantage & 3,938 & (14.5) \\
\hline Medicare, other & 1,970 & $(7.2)$ \\
\hline \multicolumn{3}{|c|}{ Duration of follow-up, months } \\
\hline Mean (SD) & 16.6 & $(17.3)$ \\
\hline Median (IQR) & 10.6 & $(5.1-21.8)$ \\
\hline$I Q R=$ interquartile range. & & \\
\hline
\end{tabular}

in the 2019 cohort had a short followup period and were excluded from the analyses). Analyses of treatment classes were not mutually exclusive and included patients who may have received multiple treatment classes. Duration of treatment was measured by time to treatment discontinuation or death (TTD), which was defined from the start of $1 \mathrm{~L}$ treatment until either death or the end of $1 \mathrm{~L}$ treatment and was analyzed using the KaplanMeier method.

\section{Results}

\section{PATIENT CHARACTERISTICS}

Between January 1, 2010, and June 30, 2019, 44,045 patients met the incident advanced-stage NSCLC algorithm criteria, of which 27,257 patients $(61.9 \%)$ met the study inclusion criteria and were included in the analysis. Patient characteristics are presented in the Table 1. Mean patient age was 62 years (median 62 years), and $52.3 \%$ of patients were male. Patients were from the southern $(31.5 \%)$, midwestern (29.3\%), western (21.4\%), and northeastern $(17.7 \%)$ regions of the United States. Patients were in the HIRD prior to NSCLC diagnosis for a mean of 41.1 months (median 35.2 months). The majority of patients were diagnosed with advanced NSCLC (74.6\%); some patients were diagnosed with early-stage NSCLC and subsequently progressed to later-stage disease (25.4\%). Overall, $78.3 \%$ of patients had commercial insurance plans, $14.5 \%$ had a Medicare Advantage plan, and $7.2 \%$ had a Medicare supplemental plan.

\section{HCRU AND COSTS DURING THE ENTIRE FOLLOW-UP PERIOD}

The mean duration of follow-up for all patients in this study was 16.6 months (median 10.6 months). Across the entire follow-up period, outpatient visits accounted for the greatest proportion of HCRU (Figure 1 and Supplementary Table 1, available in online article). Over the duration of the study, the mean number of outpatient visits was 147.1 PPPY, and the mean total number of outpatient visits over the study duration was 97.7. The mean number of hospitalizations PPPY was 2.7, and the mean length of stay PPPY was 33.4 days; the mean number of hospitalizations during the entire follow-up was 1.2, and the mean length of stay was 14.2 days.

Outpatient costs (including systemic therapy costs and other outpatient costs) constituted the majority of total costs for all patients in the study follow-up (Figure 2 and Supplementary Table 1). Mean outpatient costs for systemic anticancer therapy (ie, intravenous anticancer therapy) were $\$ 85,609$ PPPY and $\$ 61,797$ in total. Mean total costs across the entire study follow-up were \$250,942 PPPY and $\$ 158,908$ in total.

Over the duration of the study, the number of outpatient visits appeared to modestly decrease from 2010 to 2015 and then modestly increase from 2015 to 2018 (Figure 3A and Supplementary Table 2, available in online article). The number of hospitalizations, emergency department visits, and laboratory tests remained relatively stable from 2010 to 2018. Over the study duration, the total costs increased, which were driven by increasing outpatient costs related to systemic therapy (Figure 3B and Supplementary Table 2). The mean outpatient costs PPPY for systemic therapy overtook mean inpatient costs from 2015 onward and increased over the course of the study. The increase in mean outpatient costs for systemic treatments was highest in 2017 and 2018 (\$25,879 PPPY). Other costs PPPY, including inpatient costs, other outpatient costs, and pharmacy costs, remained consistent during the study period.

Analysis of treatment classes over the study duration showed that, regardless of line of treatment, chemotherapy was the most frequently used treatment (Supplementary Figure 2A, available in online article). After 2015, the proportion of patients receiving ICI-based treatments increased sharply, while the proportion of patients receiving chemotherapy 


\section{FIGURE 1 PPPY and Total HCRU During the Entire Study Follow-Up Period and the First-Line Treatment Period}
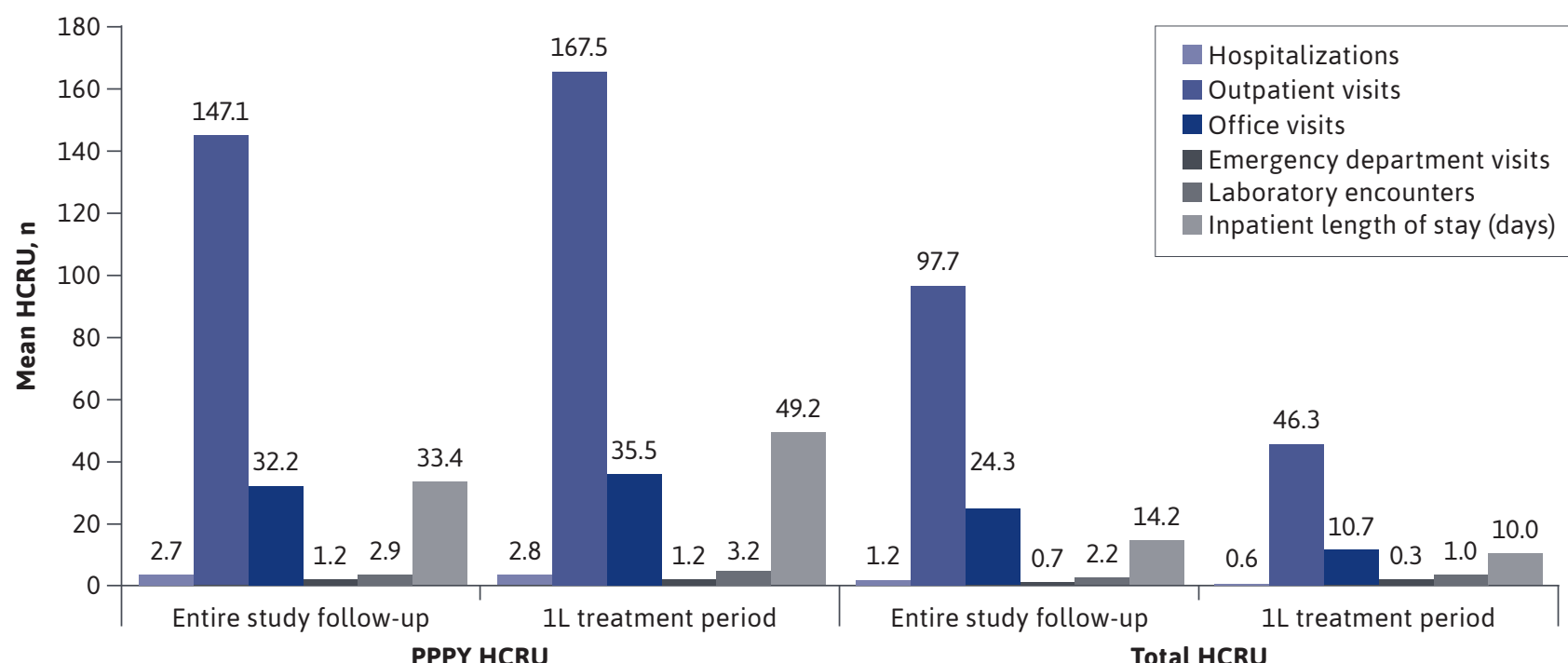

$I L=$ first line; $H C R U=$ health care resource utilization; $P P P Y=$ per patient per year.

and targeted therapy decreased. In 2018, $77 \%$ of patients received chemotherapy, 44\% received ICIs, 42\% received targeted therapies, and $10 \%$ received other treatments.

\section{HCRU AND COST DURING THE 1L TREATMENT PERIOD}

Median TTD for $1 \mathrm{~L}$ systemic therapy was 2.8 months (95\% CI not estimable; Supplementary Figure 3, available in online article).

The majority of the HCRU during the $1 \mathrm{~L}$ treatment period was for outpatient visits (Figure 1 and Supplementary Table 1). HCRU PPPY was higher during the $1 \mathrm{~L}$ treatment period vs during the entire study follow-up period, and total HCRU during 1L treatment accounted for about half of the total HCRU across the entire study follow-up (Figure 1). The mean number of outpatient visits PPPY and the mean number of total outpatient visits during the $1 \mathrm{~L}$ treatment period was 167.5 and 46.3, respectively.

The mean number of hospitalizations PPPY was 2.8, and the mean length of stay was 49.2 days. The mean number of hospitalizations during the entire follow-up was 0.6 , and the mean length of stay was 10.0 days. Outpatient costs (mean \$51,488; including outpatient systemic therapy costs and other outpatient costs) accounted for the majority of total costs during $1 \mathrm{~L}$ treatment (mean \$271,590 PPPY and $\$ 72,760$ in total; Figure 2 and Supplementary Table 1). Mean outpatient costs for systemic treatment were \$92,412 PPPY and $\$ 27,138$ in total.

During $1 \mathrm{~L}$ treatment, a trend was observed for a decreasing number of outpatient visits from 2010 to 2015, which then increased from 2015 to 2018, similar to analysis of the entire study follow-up (Figure 4A and Supplementary Table 3, available in online article). The number of hospitalizations, emergency department visits, and laboratory tests remained relatively stable from 2010 to 2018. A similar trend for increased outpatient costs from 2010 to 2018 was also seen for the $1 \mathrm{~L}$ treatment period (Figure $4 \mathrm{~B}$ and Supplementary Table 3). The mean outpatient costs for systemic therapy overtook inpatient costs from 2013 onward. The highest increase in outpatient costs for systemic therapy was between 2017 and 2018 (\$23,223 PPPY). Other PPPY costs, including inpatient costs, other outpatient costs, and pharmacy costs, were consistent across the study period.

Analyses of treatment classes during the $1 \mathrm{~L}$ treatment period were consistent with those seen in all patients in study follow-up; chemotherapy was the most frequently used treatment (Supplementary Figure 2B, available in online article). Similar to analyses of all patients in follow-up, the proportion of patients receiving ICI-based treatments increased from 2015, while the proportion of patients receiving targeted therapies decreased; the proportion of patients receiving chemotherapy was consistent 


\section{FIGURE 2 PPPY and Total Costs During the Entire Study Follow-Up Period and the First-Line Treatment Period}

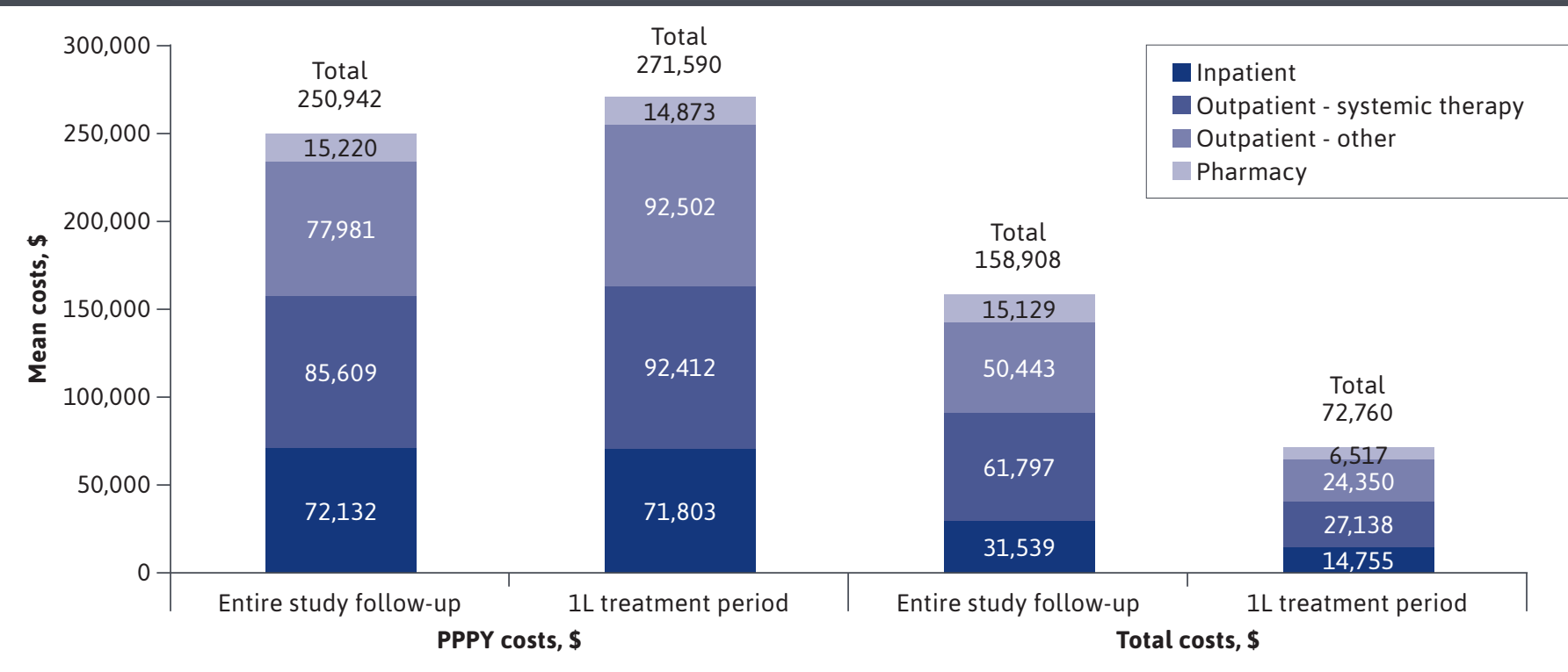

$1 L=$ first line $P P P Y=$ per patient per year .

throughout the study period. By 2018, 72\% of patients received chemotherapy, $36 \%$ received targeted therapies, $26 \%$ received ICIs, and $6 \%$ received other treatments during their first-line therapy.

\section{Discussion}

In this study, we assessed the economic burden, measured as HCRU and costs, among commercially insured patients with locally advanced or metastatic NSCLC who initiated $1 \mathrm{~L}$ systemic treatments in the United States. This manuscript reports more than 9 years of HCRU data collected from 27,257 patients, which to our knowledge, is currently the largest cohort of patients with this disease with long-term follow-up in the United States. A previous retrospective study assessing the economic burden for patients in the United States with NSCLC from 1998 to 2000 found HCRU and costs were substantial. ${ }^{24,25}$ Findings from our study nearly 20 years later confirm that locally advanced or metastatic NSCLC is still a complex disease, which despite recent improvement in treatment options, remains expensive to treat and involves considerable HCRU.

The mean costs for systemic anticancer therapy alone were approximately $\$ 86,000$ PPPY and $\$ 62,000$ in total across the entire study follow-up and approximately $\$ 92,000$ PPPY and $\$ 27,000$ in total during the $1 \mathrm{~L}$ treatment period, which were approximately $40 \%$ of the total costs. Irrespective of systemic therapy costs, the total for inpatient costs, costs for other outpatient services (preestablished definition), ${ }^{31}$ and costs for pharmacy services were an average of approximately $\$ 100,000$ in total across the entire follow-up (and approximately $\$ 165,000$ PPPY), and approximately $\$ 46,000$ in total during the $1 \mathrm{~L}$ treatment period (approximately $\$ 179,000 \mathrm{PPPY}$ ), accounting for more than $60 \%$ of the total costs.

With the recent approvals of new treatments, including ICIs from 2015 onward, the treatment landscape for NSCLC has shifted. This is reflected by the changes in treatment patterns observed over the duration of this study and other studies, which found that an increasing proportion of patients received ICI-based treatments from 2015 onward. ${ }^{33,34}$ In our study, $44 \%$ of patients received ICIs by the end of follow-up in 2018. Additionally, we noted a decline in outpatient visits from 2010 to 2014, and a sharp increase from 2015 onward. This could be attributed to a decreased utilization of chemotherapy and targeted therapies and the increased utilization of ICIs in the outpatient setting, as is seen in treatment distribution data in this study. There was some ICI use noted prior to 2015, which was most likely due to use in clinical trials.

In the previously mentioned US-based study conducted in 2005, HCRU was found to be driven by hospital inpatient 


\section{FIGURE 3 PPPY HCRU and PPPY Cost by Year During the Entire Study Follow-Up Period}

\section{A. PPPY HCRU}

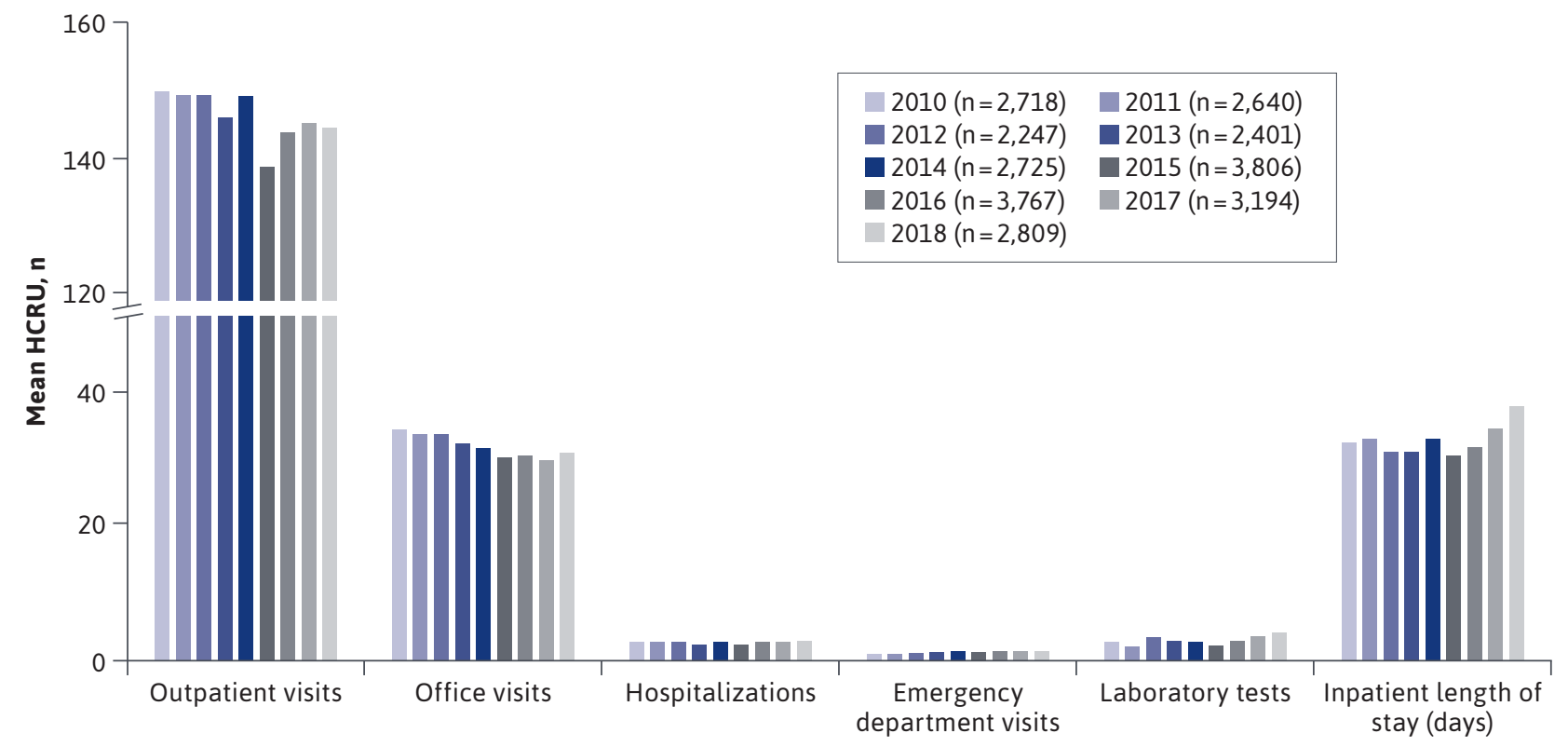

\section{B. PPPY cost}

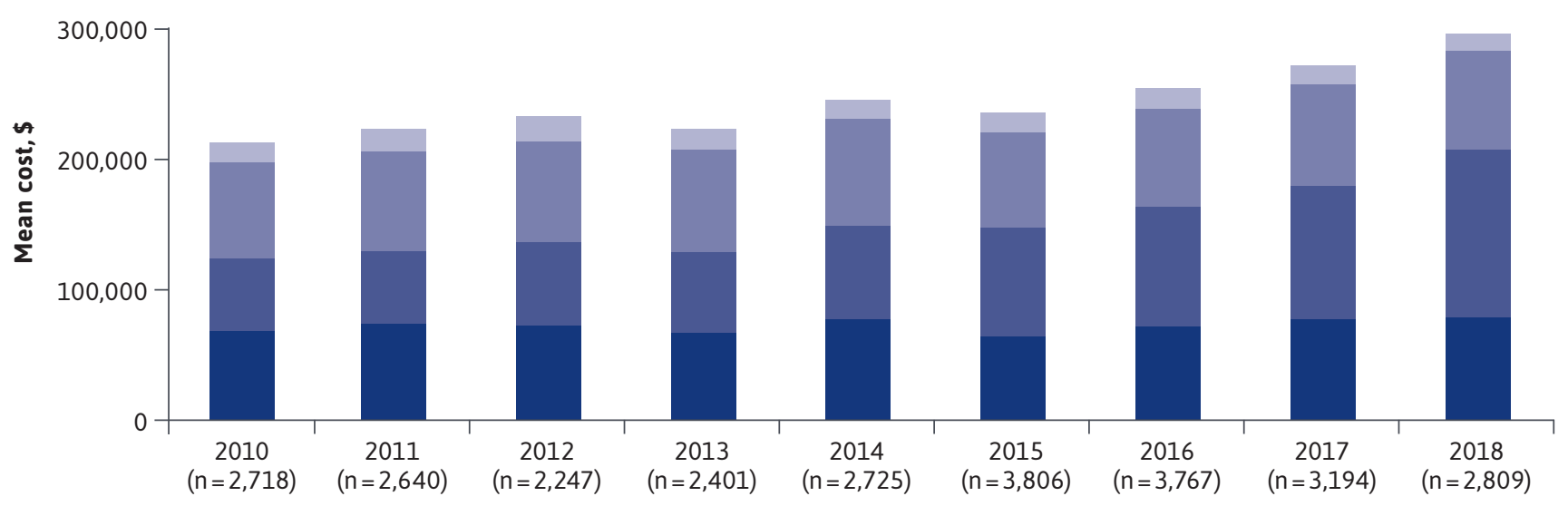

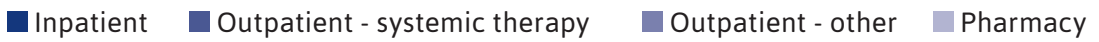

visits, including hospitalizations due to complications associated with the disease or chemotherapy. ${ }^{24}$ In our study, HCRU was found to be driven primarily by outpatient visits; consequently, outpatient costs constituted the majority of total costs for the treatment of advanced NSCLC (\$61,797 of $\$ 158,908$ total costs [38.9\%]). Additionally, outpatient costs for systemic therapy increased over the duration of the study, especially from 2015, while other costs remained relatively stable. The increase in outpatient systemic therapy costs is likely associated with the increased use of ICIs in clinical practice, which are usually billed as outpatient services. These results are consistent with findings from a previous study in a small population of patients with advanced NSCLC $(\mathrm{N}=62)$ that reported a greater outpatient 


\section{FIGURE 4 PPPY HCRU and PPPY Cost by Year During the First-Line Treatment Period}

\section{A. PPPY HCRU}

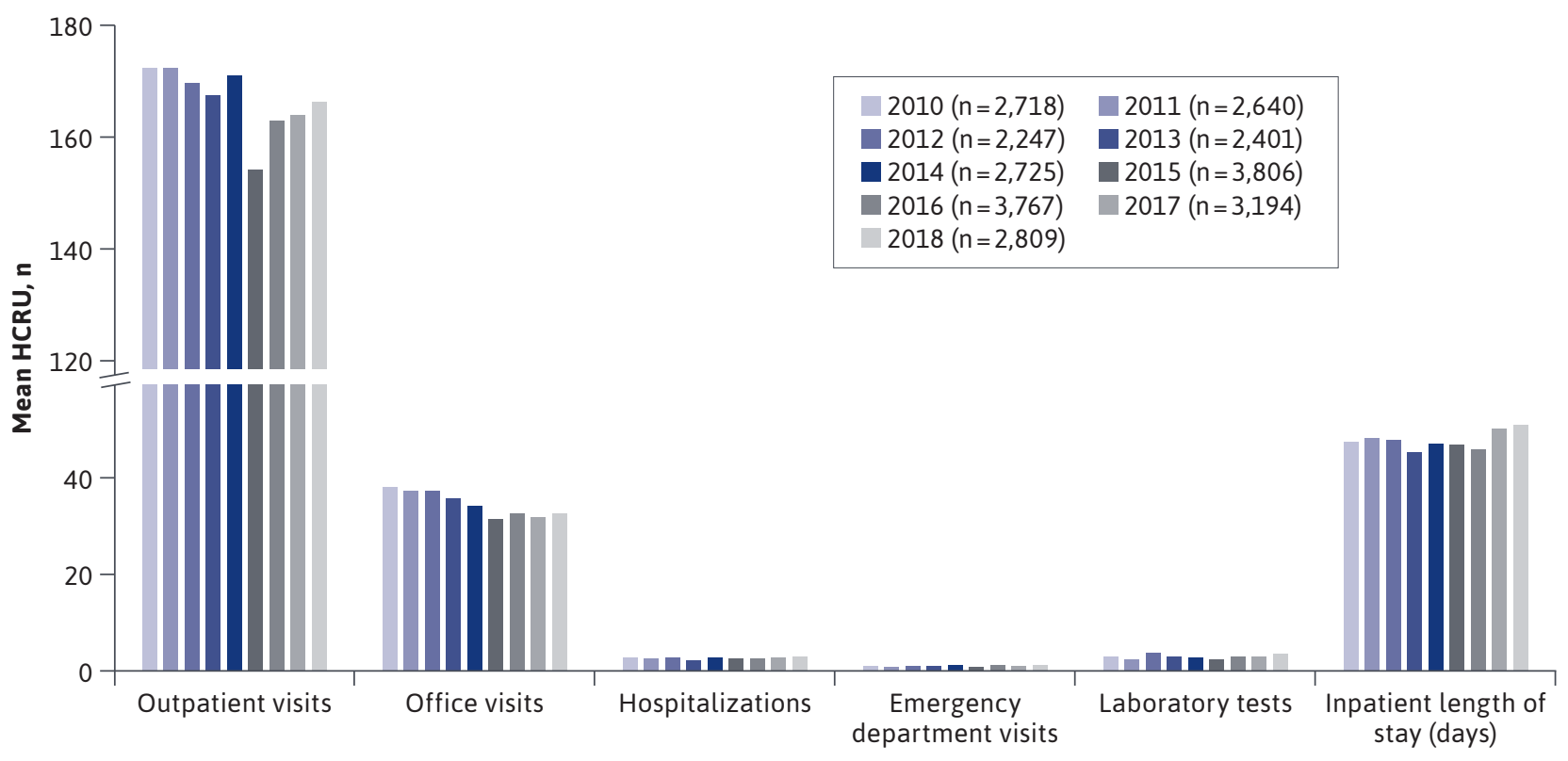

\section{B. PPPY cost}

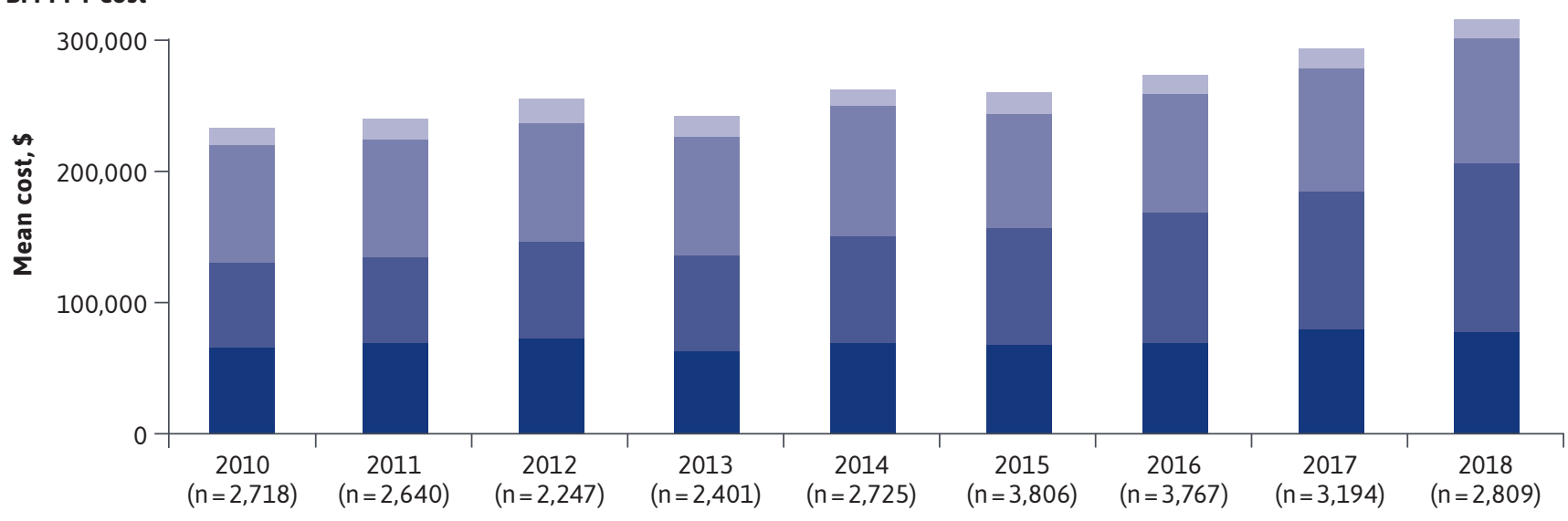

Inpatient Outpatient - systemic therapy D Outpatient - other Pharmacy

burden in analyses conducted in 2016 vs 2013 (ie, post- and preapproval of ICI treatment, respectively). ${ }^{26}$

Another study assessing the economic burden of ICIs in NSCLC conducted between 2012 and 2017 also found that the major drivers of costs for patients receiving ICIs were outpatient services, including increased treatment costs and costs for specialist office visits. ${ }^{35}$ Combined with increased treatment costs, ICIs have also been associated with a longer duration of treatment due to increased survival compared with chemotherapy in previous clinical trials, which may have further driven outpatient costs in this analysis. ${ }^{16,36-38}$

Findings from a systemic literature review assessing the economic impact of $1 \mathrm{~L}$ ICIs in NSCLC showed that 
although costs of ICI treatments are higher than those of chemotherapy, increased treatment costs appear balanced by lower HCRU and costs for AE management, including hospitalizations and emergency department visits. ${ }^{39}$ Findings from this study are corroborated by the established safety profiles of ICI monotherapies in NSCLC, and several phase 3 trials of ICI monotherapies have shown a reduced frequency and severity of AEs compared with chemotherapy in the first- and second-line setting. . $^{10,36-38,40}$

Findings from previous studies also reported that ICIs were associated with fewer AE-related hospitalizations and emergency department or ambulatory visits compared with chemotherapy. ${ }^{39,41,42}$ In this study's analysis, total number of hospitalizations, total cost of care, and total number of emergency department visits were found to be lower after the approvals of ICIs, which is consistent with previous studies. ${ }^{43}$ Although when measured via PPPY, these estimates were similar across time or increased over time. Furthermore, previous analyses of $\mathrm{AE}$ management also showed lower costs for ICIs vs chemotherapy for NSCLC when adjusted for treatment duration $(\$ 6,921$ per person per month [PPPM] vs $\$ 8,925$ PPPM; ie, $\$ 83,052$ vs $\$ 107,100$ PPPY, respectively) ${ }^{44}$ However, other studies have shown contrasting results in which ICI monotherapy (nivolumab) was associated with higher inpatient costs and total costs than chemotherapy. ${ }^{45}$ ICIs also generally resulted in higher total PPPM costs $(\$ 41,815)$ vs other systemic treatments, including chemotherapy $(\$ 24,515){ }^{46}$

Notably in this study, the hospitalization length of stay was greater during the $1 \mathrm{~L}$ period compared with the entire study follow-up. This was most likely due to patients receiving the majority of their health care during the $1 \mathrm{~L}$ treatment period and fewer patients receiving subsequent systemic treatment after discontinuation of $1 \mathrm{~L}$ treatment. Future studies of ICIs in the real-world setting are required to better understand their economic burden.

\section{LIMITATIONS}

This study has some limitations to consider. The study analyses were descriptive in nature and should be interpreted within their known limitations. As with other retrospective claim-based studies, information was lacking regarding disease histology and other clinical characteristics. The HIRD did not show all confirmed cases of advanced disease, and the analysis was reliant on a predictive model algorithm to identify further cases. Previous research has shown that the predictive model algorithm had an $80 \%$ sensitivity, $83 \%$ specificity, and a PPV of $91 \% .^{29}$

In this analysis, there was no minimum duration of follow-up. Patients who were diagnosed in the later years were associated with shorter followup, and thus the total lifetime costs were not comparable with those of patients diagnosed in earlier years; therefore, HCRU and cost data for 2019 were not included in the analyses, as a shorter follow-up period could have introduced bias. Additionally, many patients in this analysis had less than 1 year of follow-up and less than 1 year's duration of $1 \mathrm{~L}$ treatment, which resulted in PPPY HCRU and costs being numerically higher than total HCRU and costs. Considering this, analyses of PPPY HCRU and costs should be interpreted with caution.

\section{Conclusions}

The treatment landscape for advanced NSCLC has undergone a paradigm shift in recent years, with ICIs becoming new standards of care. Despite the improvement in treatment options, a substantial economic burden still exists for patients with advanced NSCLC. The increasing outpatient systemic therapy cost might reflect the greater use of ICIs for the treatment of advanced NSCLC. However, hospitalizations, other outpatient services, and pharmacy services still accounted for the majority of the economic burden. Additional approvals of ICIs occurred beyond this study period, which further highlight the pace at which the treatment landscape is evolving. These data serve as a benchmark for prospective studies of HCRU and costs associated with advanced NSCLC. Future studies are required to further investigate whether innovative treatments can increase or reduce the overall disease management cost of advanced NSCLC.

\section{DISCLOSURES}

This study was funded by the healthcare business of Merck KGaA, Darmstadt, Germany (CrossRef Funder ID: 10.13039/100009945) as part of an alliance between the healthcare business of Merck KGaA, Darmstadt, Germany, and Pfizer. Zhang, Liu, and Yang are employees of EMD Serono. Beachler, Dinh, and Jamal-Allial are employees of HealthCore Inc., which received funding from the healthcare business of Merck KGaA, Darmstadt, Germany, and Pfizer for the implementation of this study. Masters and Kolitsopoulos are employees of Pfizer. Lamy was an employee of the healthcare business of Merck KGaA, Darmstadt, Germany, at the time this study was conducted.

\section{ACKNOWLEDGMENTS}

Medical writing support was provided by Abhijith Thippeswamy of ClinicalThinking and funded by the healthcare business of Merck KGaA, Darmstadt, Germany, as part of an alliance between Merck and Pfizer.

\section{REFERENCES}

1. National Cancer Institute. Cancer stat facts: nonsmall cell lung cancer. Accessed February 24, 2021. https://seer.cancer. gov/statfacts/html/lungb.html 
2. American Society of Clinical Oncology. Lung cancer - nonsmall cell: statistics. January 2021. Accessed February 24, 2021. https://www.cancer.net/cancer-types/ lung-cancer-nonsmall-cell/statistics

3. National Comprensive Cancer Network. NCCN clinical practice guidelines in oncology. Nonsmall cell lung cancer. V5.2021. Accessed August 3, 2021.

4. Sul J, Blumenthal GM, Jiang X, He K, Keegan P, Pazdur R. FDA approval summary: pembrolizumab for the treatment of patients with metastatic nonsmall cell lung cancer whose tumors express programmed death-ligand 1. Oncologist. 2016;21(5):643-50.

5. Kazandjian D, Suzman DL, Blumenthal G, et al. FDA approval summary: nivolumab for the treatment of metastatic nonsmall cell lung cancer with progression on or after platinum-based chemotherapy. Oncologist. 2016;21(5):634-42.

6. Weinstock C, Khozin S, Suzman D, et al. FDA approval summary: atezolizumab for metastatic nonsmall cell lung cancer. Clin Cancer Res. 2017;23(16):4534-39.

7. Antonia SJ, Villegas A, Daniel D, et al. Durvalumab after chemoradiotherapy in stage III nonsmall-cell lung cancer. N Engl J Med. 2017;377(20):1919-29.

8. Reck M, Rodriguez-Abreu D, Robinson AG, et al. Pembrolizumab versus chemotherapy for PD-L1-positive nonsmall-cell lung cancer. N Engl J Med. 2016;375(19):1823-33.

9. Pai-Scherf L, Blumenthal GM, Li $\mathrm{H}$, et al. FDA approval summary: pembrolizumab for treatment of metastatic nonsmall cell lung cancer: first-line therapy and beyond. Oncologist. 2017;22(11):1392-99.

10. Spigel D, De Marinis F, Giaccone G, et al. IMpower110: interim overall survival (OS) analysis of a phase III study of atezolizumab (atezo) vs platinum-based chemotherapy (chemo) as first-line (1L) treatment (tx) in PD-L1-selected NSCLC. Ann Oncol. 2019;30(suppl 5):Abstract LBA78.
11. F. Hoffmann-La Roche Ltd. FDA approves Roche's Tecentriq as a first-line monotherapy for certain people with metastatic nonsmall cell lung cancer. May 19, 2020. Accessed April 25, 2021. https:// www.roche.com/media/releases/medcor-2020-05-19.htm

12. Sezer A, Kilickap S, Gümüş M, et al. Cemiplimab monotherapy for first-line treatment of advanced nonsmall-cell lung cancer with PD-L1 of at least 50\%: a multicentre, open-label, global, phase 3 , randomised, controlled trial. Lancet. 2021;397(10274):592-604.

13. US Food and Drug Administration. FDA approves cemiplimab-rwlc for nonsmall cell lung cancer with high PD-L1 expression. February 22, 2021. Accessed April 29, 2021. https://www.fda.gov/ drugs/drug-approvals-and-databases/ fda-approves-cemiplimab-rwlc-nonsmallcell-lung-cancer-high-pd-l1-expression

14. Socinski MA, Jotte RM, Cappuzzo F, et al. Atezolizumab for first-line treatment of metastatic nonsquamous NSCLC. N Engl J Med. 2018;378(24):2288-301.

15. US Food and Drug Administration. FDA approves atezolizumab with chemotherapy and bevacizumab for first-line treatment of metastatic non-squamous NSCLC. December 6, 2018. Accessed April 29, 2021. https://www.fda.gov/drugs/ fda-approves-atezolizumab-chemotherapy-and-bevacizumab-first-line-treatment-metastatic-non-squamous

16. West H, McCleod M, Hussein M, et al. Atezolizumab in combination with carboplatin plus nab-paclitaxel chemotherapy compared with chemotherapy alone as first-line treatment for metastatic nonsquamous nonsmall-cell lung cancer (IMpower130): a multicentre, randomised, open-label, phase 3 trial. Lancet Oncol. 2019;20(7):924-37.

17. The ASCO Post. FDA approves atezolizumab in combination with chemotherapy for first-line treatment of metastatic nonsquamous NSCLC. December 4 , 2019. Accessed April 29, 2021. https:// ascopost.com/news/december-2019/ fda-approves-atezolizumab-in-combination-with-chemotherapy-for-first-linetreatment-of-metastatic-nonsquamousnsclc/\#: :text=On\%20December\%20 4\%2C\%20the\%20U.S.,or\%20ALK\%20 genomic\%20tumor\%20aberrations
18. Gandhi L, Rodriguez-Abreu D, Gadgeel S, et al. Pembrolizumab plus chemotherapy in metastatic nonsmall-cell lung cancer. N Engl J Med. 2018;378(22):2078-92.

19. Paz-Ares L, Luft A, Vicente D, et al. Pembrolizumab plus chemotherapy for squamous nonsmall-cell lung cancer. N Engl J Med. 2018;379(21):2040-51.

20. US Food and Drug Administration. FDA grants regular approval for pembrolizumab in combination with chemotherapy for first-line treatment of metastatic nonsquamous NSCLC. August 20, 2018. Accessed April 29, 2021. https://www.fda.gov/drugs/ resources-information-approved-drugs/ fda-grants-regular-approval-pembrolizumab-combination-chemotherapy-firstline-treatment-metastatic

21. US Food and Drug Administration. FDA approves pembrolizumab in combination with chemotherapy for first-line treatment of metastatic squamous NSCLC. October 30, 2018. Accessed April 29, 2021. https://www.fda.gov/drugs/ fda-approves-pembrolizumab-combination-chemotherapy-first-line-treatmentmetastatic-squamous-nsclc

22. Paz-Ares L, Ciuleanu T-E, Cobo M, et al. First-line nivolumab plus ipilimumab combined with two cycles of chemotherapy in patients with nonsmall-cell lung cancer (CheckMate 9LA): an international, randomised, open-label, phase 3 trial. Lancet Oncol. 2021;22(2):198-211.

23. US Food and Drug Administration. FDA approves nivolumab plus ipilimumab and chemotherapy for first-line treatment of metastatic NSCLC. May 26, 2020. Accessed April 29, 2021. https://www.fda.gov/drugs/ drug-approvals-and-databases/ fda-approves-nivolumab-plus-ipilimumab-and-chemotherapy-first-line-treatment-metastatic-nsclc

24. Kutikova L, Bowman L, Chang S, Long SR, Obasaju C, Crown WH. The economic burden of lung cancer and the associated costs of treatment failure in the United States. Lung Cancer. 2005;50(2):143-54. 
25. Migliorino MR, Santo A, Romano G, et al. Economic burden of patients affected by nonsmall cell lung cancer (NSCLC): the LIFE study. J Cancer Res Clin Oncol. 2017;143(5):783-91.

26. Guo XY, Gunawan B, Sanmugarajah J. Preliminary study into the impact of immunotherapy on patterns of care and healthcare service utilization in stage IV nonsmall cell lung cancer in a tertiary hospital setting. J Clin Oncol. 2019;37(8):92.

27. Jeon SM, Kwon JW, Choi SH, Park HY. Economic burden of lung cancer: a RETrospective cohort study in South Korea, 2002-2015. PLoS One. 2019;14(2):e0212878.

28. Wood R, Taylor-Stokes G. Cost burden associated with advanced nonsmall cell lung cancer in Europe and influence of disease stage. BMC Cancer. 2019;19(1):214.

29. Esposito DB, Russo L, Oksen D, et al. Development of predictive models to identify advanced-stage cancer patients in a US healthcare claims database. Cancer Epidemiol. 2019;61:30-37.

30. Kern DM, Barron JJ, Wu B, et al. A validation of clinical data captured from a novel Cancer Care Quality Program directly integrated with administrative claims data. Pragmat Obs Res. 2017;8:149-55.

31. Find-A-Code. Berenson-Eggers Type of Service (BETOS) codes. 2011. Accessed February 24, 2021. https:// www.findacode.com/medical-code-sets/ berenson-eggers-type-of-service-betos. $\underline{\mathrm{html}}$

32. Beachler DC, Lamy F-X, Russo L, et al. A real-world study on characteristics, treatments and outcomes in US patients with advanced stage ovarian cancer. J Ovarian Res. 2020;13(1):101.
33. Nadler E, Arondekar B, Aguilar KM, et al. Treatment patterns and clinical outcomes in patients with advanced nonsmall cell lung cancer initiating first-line treatment in the US community oncology setting: a real-world RETrospective observational study. J Cancer Res Clin Oncol. 2021;147(3):671-90.

34. Leapman MS, Presley CJ, Zhu W, et al. Association of programmed cell death ligand 1 expression status with receipt of immune checkpoint inhibitors in patients with advanced nonsmall cell lung cancer. JAMA Netw Open. 2020;3(6):e207205.

35. Nesline MK, Knight T, Colman S, Patel K. Economic burden of checkpoint inhibitor immunotherapy for the treatment of nonsmall cell lung cancer in US clinical practice. Clin Ther. 2020;42(9):e1687.

36. Barlesi F, Vansteenkiste J, Spigel D, et al. Avelumab versus docetaxel in patients with platinum-treated advanced nonsmall-cell lung cancer (JAVELIN Lung 200): an open-label, randomised, phase 3 study. Lancet Oncol. 2018;19(11):1468-79.

37. Horn L, Spigel DR, Vokes EE, et al. Nivolumab versus docetaxel in previously treated patients with advanced nonsmallcell lung cancer: two-year outcomes from two randomized, open-label, phase III trials (CheckMate 017 and CheckMate 057). J Clin Oncol. 2017;35(35):3924-33.

38. Mok TSK, Wu YL, Kudaba I, et al. Pembrolizumab versus chemotherapy for previously untreated, PD-L1-expressing, locally advanced or metastatic nonsmallcell lung cancer (KEYNOTE-042): a randomised, open-label, controlled, phase 3 trial. Lancet. 2019;393(10183):1819-30.

39. Sarri G, Freitag A, Kazmierska P, Lin B, Pawar V, Zhang X. Economic impact of first-line (1L) immunotherapies in advanced/metastatic nonsmall cell lung cancer (NSCLC): a systematic literature review (SLR). Value Health. 2020;23:S443.
40. Herbst RS, Baas P, Kim DW, et al. Pembrolizumab versus docetaxel for previously treated, PD-L1-positive, advanced nonsmall-cell lung cancer (KEYNOTE-010): a randomised controlled trial. Lancet. 2016;387(10027):1540-50.

41. Engel-Nitz N, Ryan K, Johnson MP, Bunner S. Health care utilization associated with adverse events (AEs) among metastatic nonsmall cell lung cancer (mNSCLC) patients treated with immunotherapy or chemotherapy. J Clin Oncol. 2019;37(15):e20655.

42. Radtchenko J, Korytowsky B, Nwokeji E, Tuell K, Feinberg BA. Hospitalizations and emergency department (ED) visits pre- and post-approval of immuno-oncology agents (IO) in advanced nonsmall cell lung cancer (aNSCLC). J Clin Oncol. 2018;36(15):e21006.

43. Korytowsky B, Radtchenko J, Nwokeji ED, Tuell KW, Kish JK, Feinberg BA. Understanding total cost of care in advanced nonsmall cell lung cancer preand postapproval of immuno-oncology therapies. Am J Manag Care. 2018;24(20):S439-47.

44. Stenehjem DD, Santi I, Gupte-Singh K, Penrod JR, Venkatachalam M. Estimated costs of grade 3-4 all-cause and treatment-related adverse events for stage IV or recurrent nonsmall cell lung cancer (NSCLC) in the CheckMate 227 (CM-227) trial. J Clin Oncol. 2019;37(8):120.

45. Newcomer LN, Byfield SD, Chastek B, Korrer S, Horstman T, Malin J. Describing the value of the most common first line NSCLC regimens in a real world setting. J Clin Oncol. 2017;35(15):9046.

46. Cohen JT, Lin PJ, Sheinson DM, et al. Are National Comprehensive Cancer Network Evidence Block affordability ratings representative of real-world costs? An evaluation of advanced nonsmall-cell lung cancer. J Oncol Pract. 2019;15(11):e948-56. 